\title{
Saturation of the anomalous Kapitza conductance
}

\author{
H. Schubert, P. Leiderer, and H. Kinder \\ Physik Department der Technischen Universität München, D-8046 Garching, West Germany
}

(Received 24 May 1982)

\begin{abstract}
The reflection coefficient of longitudinal $25-\mathrm{GHz}$ phonons was measured at normal incidence on quartz-liquid ${ }^{4} \mathrm{He}$ interfaces. For clean samples, close agreement with the Khalatnikov theory was found with no indication of anomalous transmission. Samples coated with $300-\AA$ paraffin, however, showed a substantially lower reflection coefficient in contact with helium. This anomalous reduction was found to depend on the phonon intensity $I$ as $I^{-1 / 2}$, in good accord with the defect model for the Kapitza conductance.
\end{abstract}

The thermal transport across the interface between solids and liquid helium has been a long standing problem. ${ }^{1}$ As a result of the mismatch of the acoustic impedances of liquid helium and usual solids the transmission coefficient for phonons in general ought to be less than $1 \% .^{2}$ Experimentally, however, transmission coefficients one or two orders of magnitude larger have been observed. ${ }^{3}$ Most attempts to explain this discrepancy had been based on the assumption of an ideally flat and clean surface, until experiments of phonon reflection at freshly cleaved surfaces ${ }^{4}$ revealed a drastic reduction of the anomalous Kapitza conductance. Two recent suggestions hence have taken surface irregularities into account: one assumes surface roughness, ${ }^{5}$ and the other one defects, ${ }^{6}$ viz., "two-level systems" (TLS) to be the cause for the anomalous heat transport. For the defect model, saturation of the TLS at high phonon intensities is expected like in bulk glasses, ${ }^{7}$ while there are no saturation effects expected for the roughness model. In fact, we have found a qualitative influence of the phonon intensity in a previous experiment. $^{8}$ In this Communication, we present quantitative results on the intensity dependence of the reflection coefficient at a quartz-liquid-helium interface. The results strongly support the defect model and allow us to extract the coupling constant of the defects with helium.

The phonons were generated in a thin quartz platelet by means of stimulated Brillouin scattering using a giant pulse ruby laser, as described previously. ${ }^{8}$ The monochromatic longitudinal phonons obtained in this way have a wavelength $\lambda_{\mathrm{ph}}=\lambda_{0} / 2 n$, where $\lambda_{0}$ is the wavelength of the laser light in vacuum, $6943 \AA$, and $n$ is the refractive index of quartz. For $x$ quartz, this corresponds to a phonon frequency of $24.8 \mathrm{GHz}$. These phonons were reflected back and forth between the parallel surfaces of the platelet at nearly normal incidence. On every other reflection, the phonons were detected by means of a second laser with lower power. Since the bulk attenuation is negligible here, the reduction in the intensity of suc- cessive echoes is $(1-R)^{2}$, where $R$ is the reflection coefficient. The temperature for all experiments reported here was $1.8 \mathrm{~K}$.

First we discuss results obtained with a sample thoroughly cleaned with acetone. In this case $R$ turned out to be close to unity. Hence, for maximum accuracy we have normalized each echo obtained with the sample in helium by the corresponding one without helium. This is shown in the upper trace of Fig. 1, for a sample covered with a saturated helium film of $350-\AA$ thickness. The best fit of the reflection coefficient to the data is $R_{\text {expt }}=0.992$ \pm 0.003 . This result agrees exceedingly well with the value $R_{\mathrm{AM}}=0.992$, expected according to the acoustic mismatch theory of Khalatnikov. ${ }^{2}$ Thus our measurements show for the first time that for longitudinal phonons at nearly normal incidence the Khalatnikov model is valid for frequencies at least up to $25 \mathrm{GHz}$. A dependence of the reflection coefficient on the phonon intensity was not observed in this case, nor

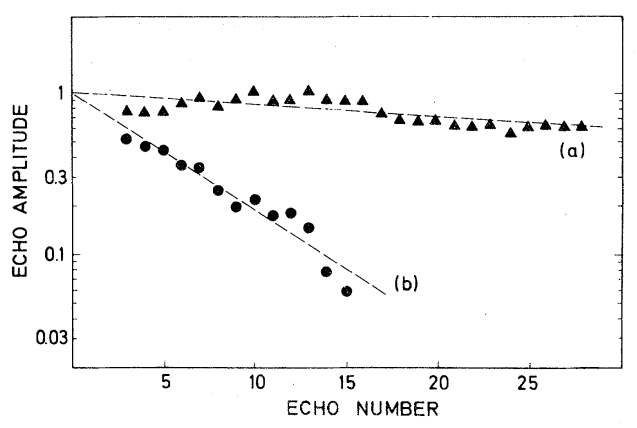

FIG. 1. Normalized echo amplitudes of a phonon pulse $(\nu=24.8 \mathrm{GHz})$ successively reflected in a quartz platelet at normal incidence. Trace (a), cleaned sample surface; trace (b), surface coated with a 300 - $\AA$ layer of paraffin, low phonon intensity $\left(5 \times 10^{-3} \mathrm{~W} / \mathrm{cm}^{2}\right)$; and dashed lines, leastsquares fits with reflection coefficients of $0.992 \pm 0.003$ and $0.92 \pm 0.01$, respectively. The time between two echoes, corresponding to one round trip of the phonons was $120 \mathrm{~ns}$ in the platelet of $0.35-\mathrm{mm}$ thickness. 
was it expected on the basis of the acoustic mismatch theory. We have also investigated the dependence of the Khalatnikov transmission on the thickness $d$ of the helium film. Below $d \simeq 100 \AA$ the transmission started to decrease and disappeared at $d \simeq 10 \AA$. For comparison, the wavelength in helium is $100 \AA$.

Our main concern was not the Khalatnikov behavior, however, but rather the anomalous transmission. As shown by Sabisky and Anderson, ${ }^{9}$ the latter can be greatly enhanced by exposing the sample to "pump oil vapors." For more reproducible conditions, we have therefore evaporated homogeneous films of paraffin onto both sides of the samples. Typically, these films were $300 \AA$ thick $\left(\lambda_{\text {ph }} \simeq 1000\right.$ $\AA$ in paraffin) as measured with a quartz gauge. In principle there would be no surprise if the hydrocarbon layer gave rise to considerable additional damping. Yet the reflection coefficient was found to be unchanged within an absolute accuracy of $1 \%$, as long as the sample was exposed to vacuum, indicating that the paraffin layer alone has no noticeable effect on the specular reflection of $25-\mathrm{GHz}$ phonons. A pronounced drop in $R$ was found, however, when helium was added to the coated quartz surface, as seen in the lower trace of Fig. 1. Thus the paraffin mediates the interaction between the phonons and the helium.

The problem of interest is now how the decrease of the reflection coefficient depends on the phonon intensity. Our results showing this dependence are plotted in Fig. 2 for phonon intensities varying over three orders of magnitude. An unsaturated helium film of $d \simeq 10 \AA$ was used here to suppress the Khalatnikov transmission. The saturation observed at high intensities is reminiscent of the ultrasonic absorption in amorphous solids where the attenuation of phonons is ascribed in part to the resonant absorption of TLS with a broad distribution of excitation

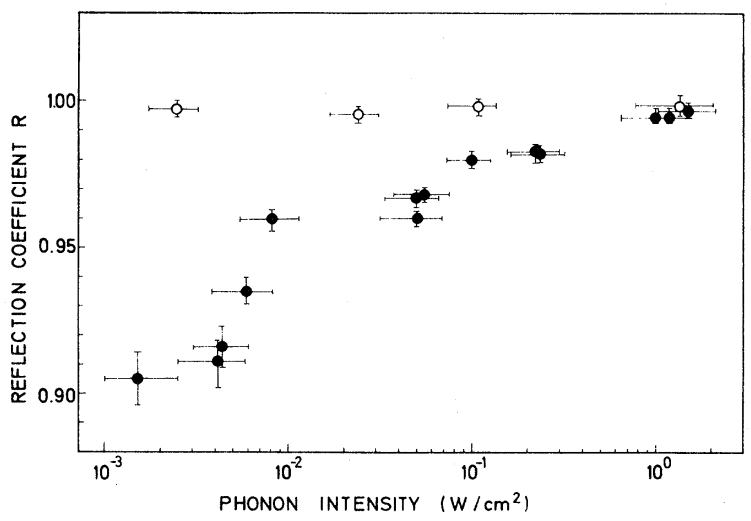

FIG. 2. Absolute phonon reflection coefficient of a paraffin-coated quartz sample as in Fig. 1(b), as function of the phonon intensity. Full symbols, in contact with helium and open symbols, without helium. energies $^{7}$ :

$$
\alpha=\alpha_{0} \tanh (\hbar \omega / 2 k T) /\left(1+I / I_{c}\right)^{1 / 2},
$$

where $\alpha_{0}$ expresses the attenuation at temperature $T=0$ and intensity $I=0$, and the additional factor describes the population dependence with the critical intensity $I_{c}$. At high intensities the absorption follows an $I^{-1 / 2}$ law. In our case, $\alpha$ corresponds to $1-R$. To compare the data of Fig. 2 with the $I^{-1 / 2}$ law, we have plotted $\log _{10}(1-R)$ in Fig. 3. In fact, there is close agreement at high intensities.

At very low intensities, $1-R$ should become constant according to (1). Unfortunately, this range was not accessible within the restricted sensitivity of our experiment. However, the deviation of the data in Fig. 3 from the straight line, apparent at the lowest intensities, might be an indication for the onset of this constant regime. Although an accurate determination appears to be difficult, the data at least allow one to give an upper limit for the critical intensity, $I_{c} \leqslant 1 \mathrm{~mW} / \mathrm{cm}^{2}$. From the critical intensitity, the relaxation rate $\tau^{-1}=\tau_{\mathrm{He}}^{-1}+\tau_{\mathrm{sol}}^{-1}$ of the TLS can be obtained $^{10}$ :

$$
I_{c}=\left(\hbar^{2} \rho_{\mathrm{sol}} v_{\mathrm{sol}}^{3} / 2 M_{\mathrm{sol}}^{2}\right) \tau^{-2} .
$$

Here $\rho_{\text {sol }}=0.95 \mathrm{~g} / \mathrm{cm}^{3}$ and $v_{\text {sol }}=1.4 \times 10^{5} \mathrm{~cm} / \mathrm{s}$ are the density and longitudinal sound velocity of paraffin, ${ }^{11}$ and $M_{\text {sol }}$ is the deformation potential of the TLS due to deformations of the solid. Typically, $M_{\text {sol }} \simeq 1 \mathrm{eV}$ is found for glassy materials. Taking these values, we find as an upper limit: $\tau^{-1}$ $\leqslant 10^{10} \mathrm{~s}^{-1}$

As long as there is no helium on the surface, the excited TLS can relax only via $\tau_{\text {sol. }}^{-1}$ The fact that no attenuation was observed without helium even for the lowest intensities implies that $\tau_{\text {sol }}^{-1}$ is small so that $I_{c}<<I$, and $\alpha \rightarrow 0$ according to (1). As soon as helium is added to the surface, the TLS can relax also via the second channel, at the rate $\tau_{\mathrm{He}}^{-1}$. The fact that attenuation was indeed observed in this case implies

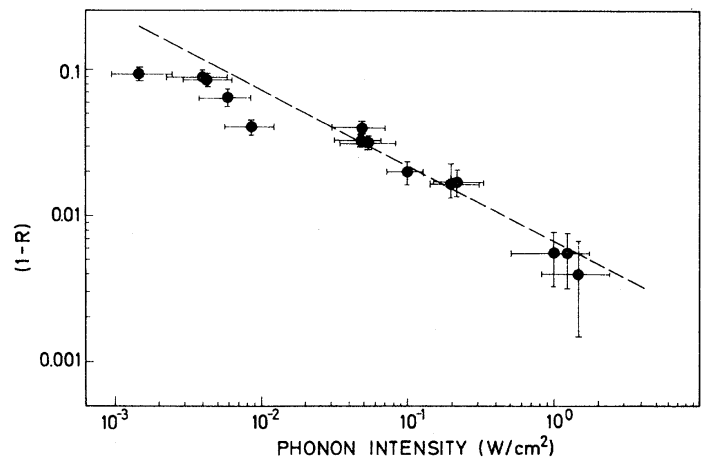

FIG. 3. Data of Fig. 2 plotted on a $\log -\log$ scale. The dashed line has a slope of $-\frac{1}{2}$. 
that $\tau_{\mathrm{He}}^{-1} \gg \tau_{\mathrm{sol}}^{-1}$ so that $I_{c}$, and hence $\alpha$ is increased. Therefore, we have $\tau_{\mathrm{He}}^{-1} \simeq \tau^{-1}$ and also $\tau_{\mathrm{He}}^{-1} \leqslant 10^{10} \mathrm{~s}^{-1}$.

On the other hand, the emission rate into the liquid $\mathrm{He}$ can be expressed by ${ }^{6}$

$$
\tau_{\mathrm{He}}^{-1}=\left(M_{\mathrm{He}}^{2} \omega^{3} / 2 \pi \hbar \rho_{\mathrm{He}} v_{\mathrm{He}}^{5}\right) \operatorname{coth}(\hbar \omega / 2 k T) .
$$

Using $\rho_{\mathrm{He}}=0.146 \mathrm{~g} / \mathrm{cm}^{3}$ and $v_{\mathrm{He}}=2.4 \times 10^{4} \mathrm{~cm} / \mathrm{s}$ we thus obtain a lower limit for the deformation potential of the defects by the helium density fluctuations: $M_{\mathrm{He}} \leqslant 2 \mathrm{meV}$. This is just the order of magnitude expected for a coupling via van der Waals forces between the defect atom and the surrounding helium. ${ }^{6}$

Thus, the present data on paraffin coated surfaces with phonons at nearly perpendicular incidence demonstrate that the energy transfer between solids and liquid helium is mediated by saturable defects in this case. Also, the deformation potentials give quantitative support to the defect model. In the more general situation of oblique incidence, the phonon strain is much larger at the surface ${ }^{6}$ so that less defects are required for the same absorption probability. On "normal" surfaces, the natural abundance of surface defects like steps, kinks, adatoms, adsorbed molecules, or layers of ice may hence be sufficient to explain the anomalous Kapitza conductance by the same mechanism.

\section{ACKNOWLEDGMENT}

We appreciate valuable discussions with W. Dietsche.
1P. L. Kapitza, Zh. Eksp. Teor. Fiz. 11, 1 (1941).

${ }^{2}$ I. M. Khalatnikov, Zh. Eksp. Teor. Fiz. 22, 687 (1952).

${ }^{3}$ For recent reviews, see, e.g., A. F. G. Wyatt, in Nonequilibrium Superconductivity, Phonons, and Kapitza Boundaries, edited by K. E. Gray (Plenum, New York, 1981), p. 31, and the chapter on Kapitza Resistance, in Phonon Scattering in Condensed Matter, edited by H. J. Maris (Plenum, New York, 1980), pp. 173-242.

${ }^{4}$ J. Weber, W. Sandmann, W. Dietsche, and H. Kinder, Phys. Rev. Lett. 40, 1469 (1978).

${ }^{5}$ N. S. Shiren, Phys. Rev. Lett. 47, 1466 (1981).
${ }^{6}$ H. Kinder, Physica (Utrecht) B 107, 549 (1981).

${ }^{7}$ S. Hunklinger and W. Arnold, in Physical Acoustics, edited by W. P. Mason and R. N. Thurston (Academic, New York, 1976), Vol. 12, p. 155.

${ }^{8} \mathrm{H}$. Schubert, P. Leiderer, and H. Kinder, J. Low Temp. Phys. 39, 363 (1980).

${ }^{9}$ E. S. Sabisky and C. H. Anderson, Solid State Commun. 17, 1095 (1975).

${ }^{10}$ From Ref. 7, neglecting "spin-spin" interaction.

${ }^{11}$ E. Irons, J. Sci. Instrum. 7 323 (1930). 